Serrano Rodríguez, R. y Pontes Pedrajas, A. (2017). Diferencias entre Expectativas y Logros en las Competencias del Prácticum del Máster de Formación del Profesorado de Enseñanza Secundaria. Revista Electrónica Interuniversitaria de Formación del Profesorado, 20(1), 1-18.

\title{
Diferencias entre Expectativas y Logros en las Competencias del Prácticum del Máster de Formación del Profesorado de Enseñanza Secundaria
}

Rocío Serrano Rodríguez, Alfonso Pontes Pedrajas

Universidad de Córdoba (España)

\section{Resumen}

Conocer las expectativas sobre el módulo de Prácticum del Máster Formación del Profesorado de Enseñanza Secundaria (FPES) y las opiniones sobre el desarrollo de competencias en dicho modulo es la meta fundamental de esta investigación. Por ello hemos encuestado a 353 alumnos y alumnas del Máster de la Universidad de Córdoba, usando un cuestionario de escala Likert que nos ha permitido comparar las opiniones sobre la importancia atribuida y el desarrollo alcanzado en tales competencias. Los resultados muestran diferencias significativas entre sus opiniones sobre tales aspectos, poniendo de manifiesto algunos logros y deficiencias del proceso formativo. Creemos que estos resultados son útiles para seguir avanzado en la investigación sobre el pensamiento inicial docente y desarrollar propuestas fundamentadas que contribuyan a mejorar la formación inicial del profesorado de secundaria en el futuro.

\section{Palabras clave}

Máster de profesorado de enseñanza secundaria; opiniones del alumnado; expectativas sobre la formación en centros; competencias desarrolladas. 


\title{
Differences between Expectations and Achievements in the Competences of the Practicum module of the Master's Degree in Secondary Teacher Training
}

\begin{abstract}
This project has been carried out in order to know the expectations about the Practicum module of the Master's Degree in Secondary Teacher Training, with the main aim of knowing the opinions about the development of skills in this module. 353 students attending this Master at the University of Cordoba have been surveyed with a Likert scale questionnaire after the development of teaching practices in secondary schools. Results show significant differences among their expectations and achievements in the competences of the Practicum. We believe that these results are useful to improve the understanding of the opinions of students, identifying their training needs and thoughts and in order to develop proposals to improve the initial training of secondary school teachers in the future.
\end{abstract}

\section{Key words}

Master's Degree in Secondary Teacher Training; opinions of students; expectations about the training in the school; developed competences.

\section{Introducción}

La implantación del Máster de Formación del Profesorado de Enseñanza Secundaria (MFPES) en nuestro país, supuso un aumento del interés por la investigación educativa en torno a la formación inicial de los futuros profesores y profesoras de esta etapa. Una de las líneas de investigación que ha cobrado auge es el estudio del pensamiento inicial de los futuros docentes (Rodríguez y Díez, 2014) analizando sus expectativas y la visión de los logros alcanzados en torno a las competencias concretas previstas en el citado máster (Solís, Rivero y Porlán, 2013). En este contexto hemos desarrollado algunos estudios sobre las demandas formativas, el desarrollo de competencias y la construcción de la identidad profesional docente (Pontes y Serrano, 2010; Pontes Serrano, 2013), que nos han servido de base para profundizar en esta línea de investigación y plantearnos nuevos interrogantes para seguir avanzando. Al desarrollar este nuevo estudio estamos interesados en explorar las opiniones de los estudiantes sobre las competencias profesionales desarrolladas durante la fase práctica del máster FPES, distinguiendo entre el nivel de desarrollo y la importancia que se les concede a tales competencias para mejorar la profesionalidad docente, con vistas a conocer las fortalezas y debilidades del proceso actual de formación inicial docente y formular propuestas bien fundamentadas que ayuden a mejorar este proceso (Martín, Prieto y Lupión, 2014).

\section{Fundamento}

Los futuros profesores y profesoras de secundaria poseen actitudes, ideas y creencias sobre la profesión docente y las necesidades formativas del profesorado de 
educación secundaria, que influyen en el desarrollo de la profesionalidad docente (Mellado, Blanco y Ruiz, 1999; Bolívar, 2006; Manso y Martín, 2013). El estudio de las expectativas, opiniones y reflexiones personales sobre los procesos educativos que realizan los futuros profesores y profesoras, durante el proceso de formación inicial, tiene gran importancia para conocer su pensamiento docente, para desarrollar un modelo reflexivo de formación (Schön, 1992; Perrenoud, 2001), para diseñar estrategias y recursos que ayuden a mejorar dicha formación y también es útil para mejorar la formación de los propios profesorestutores del prácticum (Gutiérrez et al., 2003).

En varios estudios previos sobre el proceso de formación inicial (Pontes y Serrano, 2010; Pontes, Serrano y Poyato, 2013) la mayoría de los sujetos contemplan la docencia en educación secundaria como una salida profesional importante o necesaria, realizando el curso de formación inicial por motivos de pragmatismo fundamentalmente, aunque también hay una minoría importante que lo hace por vocación o por interés en mejorar sus conocimientos docentes. En su mayor parte los aspirantes a profesores presentan una visión un poco pesimista de la docencia en educación secundaria, por el predominio de estereotipos relacionados con los conflictos que surgen en los centros de secundaria o la desmotivación del alumnado de esta etapa. Pero la mayoría de los futuros docentes asumen la necesidad de disponer de formación docente específica para ser profesor de enseñanza secundaria en la actualidad, debido a los problemas de la educación en la sociedad moderna y a la complejidad de esta profesión en la actualidad. Entre los principales elementos que consideran que deben formar parte de ese proceso de formación señalan aspectos generales relacionados con la capacidad de resolver conflictos, adquirir técnicas de comunicación, aprender a programar la enseñanza y saber fomentar la motivación de los alumnos por el aprendizaje, haciendo hincapié en la importancia de adquirir tales conocimientos a través de una formación eminentemente práctica.

A partir de tales resultados hemos observado la necesidad de seguir explorando estas ideas previas, centrando nuestra atención en analizar sus opiniones sobre las competencias docentes tras el desarrollo de la fase práctica del curso de formación inicial, que supone un primer encuentro con la realidad de la actividad docente en los centros de educación secundaria. Aunque la problemática del prácticas del máster FPES no es igual a la de los profesores noveles o principiantes, porque se trata de alumnos y alumnas que realizan una primera y breve aproximación al ejercicio de la función docente, bajo la tutela y supervisión de un profesor-tutor en el centro de prácticas, pensamos que las concepciones, actitudes, temores y esperanzas que manifiestan en sus reflexiones sobre la enseñanza, tienen bastante relación con la problemática de la formación de profesores principiantes (Rodríguez, 2003).

Una línea de trabajo importante en torno a esta temática es el análisis de las ideas y reflexiones que los futuros profesores de enseñanza secundaria muestran en los informes de prácticas (Zabalza, 2004; Solís, Porlán y Rivero, 2012). Muchos de estos informes o memorias (proyectos de prácticas, artículos y estudios sobre dicho tema, etc.) siguen siendo proposiciones bien intencionadas pero carentes de datos que les sirvan de soporte. En general reflejan opiniones, valoraciones personales, ecos de la propia experiencia informal, pero aportan pocos datos acerca de la realidad educativa. En un trabajo anterior (Pontes y Serrano, 2008), comenzamos a interesarnos por esta temática realizando un análisis cualitativo de algunos aspectos reflejados en las memorias de prácticas de los 
alumnos del curso del CAP. Los datos recogidos nos permitieron comprobar que las prácticas del curso de formación inicial eran satisfactorias para la mayoría del alumnado, observando que disminuyeron algunos prejuicios sobre los problemas de disciplina o convivencia y que muchos sujetos experimentaron una mejora en su actitud hacia la profesión docente y hacia el contexto educativo de la educación secundaria actual, aunque reconocían la necesidad de seguir mejorando su formación didáctica y desarrollando competencias docentes de tipo práctico.

La formación inicial docente basada en competencias es un reto complicado para los formadores y formadoras del profesorado (Gairín, 2011), constituyendo actualmente una línea de trabajo de gran importancia (Gutiérrez, 2011; Rodríguez et al., 2013). En un estudio sobre esta temática, realizado con alumnado del Máster FPES en la Universidad de Valladolid (Torrego, 2013), los estudiantes afirman que el periodo de prácticas ha sido en el que más han aprendido y de una forma más satisfactoria que en la fase teórica. Concretamente el $83 \%$ de los encuestados señalan haber conocido la estructura, organización y funcionamiento del centro, afirmando en un $67 \%$ que han podido aplicar los conocimientos teóricos adquiridos en el Máster FPES. En consonancia estos datos con los resultados obtenidos en la investigación desarrollada en la Universidad de Zaragoza (Cano, Orejudo y Cortés. 2012), donde la opinión global del alumnado sobre la formación recibida y las competencias adquiridas durante el Prácticum es bastante positiva, aunque se aprecian necesidades formativas en TICs e innovación curricular (Valverde, Garrido y Fernández, 2010; Ramírez, Sánchez, García y Latorre, 2011).

Sin embargo, debemos de tener en cuenta las aportaciones del trabajo realizado por Zabalza (2011) sobre el estado de la cuestión del Prácticum en la formación superior, donde analiza siete aspectos que considera fundamentales en el desarrollo del Prácticum. De entre los siete destacamos el componente emocional de la experiencia del Prácticum. Cuando se analizan los diarios de prácticas, resulta bastante habitual descubrir, por lo general, que los y las estudiantes se sienten felices afirmado que están aprendiendo mucho. Sin embargo, caen con frecuencia en la tentación de desmerecer lo que han aprendido en las aulas porque tiene poco que ver (o ellos no ven la relación) con lo que ellos están haciendo y viviendo (Hascher, Cocard y Moser, 2004). Por ello, autores como Sarmiento, Cid y Pérez (2011) proponen la necesidad de mejorar la evaluación, la acreditación y la concreción de las competencias en el Prácticum del Máster FPES mediante la implementación de instrumentos de evaluación interdisciplinares y basados en estándares de calidad (Cebrián, 2011; Zabalza, 2011; Albert, Fuentes y Palos, 2012).

Pérez-Valiente (2011) profesor de Educación Secundaria hace un aproximación sobre cuáles deberían ser los criterios de calidad reales para abordar el Prácticum del Máster FPES. Apunta hacia la necesidad de posibilitar a los y las estudiantes competencias personales y profesionales sobre: formación en didácticas actualizadas, en acción tutorial (Cid, Pérez y Sarmiento, 2011), en organización y gestión del aula y del centro educativo. Otros autores como Álvarez, Rodríguez, González, et al. (2010), se centran en la línea de competencias docentes básicas (programación de la enseñanza, diseño de unidades didácticas, desarrollo de actividades en el aula, evaluación del aprendizaje,...) y otras competencias complementarias (prevención de la violencia escolar y la mejora de la convivencia entre los miembros de la comunidad educativa). Armengol, Castro, Jariot y otros (2011), tras el estudio desarrollo en la Universidad Autónoma de Barcelona apuntan la 
necesidad de incorporar competencias socioemocionales y de gestión en los profesionales de la educación. En otros estudios dentro del contexto del Prácticum de los futuros y futuras maestras y maestros (Mendoza, 2013), los hallazgos revelaron una marcada tendencia a percibir que la institución formadora (universidad) y su agente más representativo (tutor o tutora) no responden a las demandas de apoyo emocional, profesional y pedagógico.

Otros autores (Coll, 2010; Feito, 2010; Pérez, 2010), apuestan por la implementación de competencias vinculadas al conocimiento de la cultura organizacional de los Centros de Educación Secundaria, como parte esencial de un proceso de cambio en beneficio del Prácticum del Máster FPES (González Sammaned, 2009; Morales, Narváez y Morales, 2012). Si bien, la fase práctica del citado máster prevé un periodo de observación y otro de intervención y experimentación, en muchos programas los y las estudiantes pasan la mayor parte del tiempo observando sin experimentar, intervenir, innovar o dar clases (Puentes, 2013). En aquellos casos en los que el alumnado realmente experimenta y da clases, le resulta difícil efectuar verdaderas innovaciones pedagógicas (González Sanmamed y Abeledo, 2011: Valdés y Bolívar, 2014).

Tras la revisión realizada consideramos necesario explorar las opiniones del profesorado en formación sobre las competencias profesionales desarrolladas en el Máster FPES, analizando las fortalezas y debilidades del proceso como base para el diseño de propuestas fundamentadas que ayuden a mejorar la formación docente en el futuro (Vázquez y Ortega, 2011).

\section{Metodología y Diseño de la Investigación}

\section{Objetivos}

En este estudio pretendemos conocer las opiniones de los estudiantes del máster FPES sobre la importancia formativa que conceden a las competencias profesionales del Prácticum y el grado de desarrollo de las mismas que creen haber alcanzado durante el periodo de prácticas en centro docentes. Se trata también de identificar las debilidades y fortalezas del citado máster, en sus primeros años de implantación, con vistas a formular una propuesta fundamentada de mejora de la FIPS. Dentro de este enfoque global se pretenden desarrollar los objetivos específicos siguientes:

1. Explorar las expectativas del alumnado del máster FPES sobre el módulo Prácticum, analizando la importancia formativa que los estudiantes otorgan a las competencias profesionales que se deberían desarrollar en dicho módulo.

2. Analizar las opiniones de los futuros y futuras docentes sobre el nivel de desarrollo de las competencias del Prácticum, para conocer las posibles deficiencias del proceso de formación inicial docente.

3. Comparar las diferencias entre expectativas y logros relativos a tales competencias como medio para identificar aspectos del proceso a los que hay que prestar más atención y que sirvan para formular propuestas de mejora del nuevo modelo de formación del profesorado de secundaria. 


\section{Participantes}

La muestra de investigación está constituida por 353 estudiantes del Máster FPES de la Universidad de Córdoba en los cursos 2009-2010 y 2010-2011, con una edad media de 25.7 años y una ligera mayoría de mujeres (53,8\%) sobre hombres. Esta muestra se distribuye en cuatro macro-áreas de conocimiento: Ciencias Experimentales (31,5\%), Ciencias Instrumentales (30\%), Ciencias Sociales $(20,4 \%$ ) y Humanidades $(18,1 \%)$.

\section{Instrumentos de investigación y recogida de datos}

Con el fin de conocer las expectativas y la valoración en la adquisición de las competencias vinculadas al módulo de "Prácticum", los estudiantes respondieron a un cuestionario Likert con una escala de valoración que incluye cinco categorías posibles: de 1 (mínimo) a 5 (máximo). Los datos fueron recogidos durante el desarrollo del curso académico mediante la Plataforma Moodle en un seminario virtual incluido de la asignatura "Prácticas en centro docentes". El alumnado cumplimentaba el cuestionario a través de una tarea planificada que debían adjuntar conjuntamente a la entrega de su memoria.

El cuestionario incluye una sección inicial de datos socio-demográficos (edad, género, año de finalización de estudios y especialidad del máster). Las preguntas siguientes se organizan en dos partes de tres secciones cada una, en la que se han de valorar los objetivos (A), las competencias generales (B) y las competencias establecidas para el Prácticum (C) del máster FPES. En la $1^{\mathrm{a}}$ parte se trata de valorar el grado o nivel de desarrollo alcanzado en tales aspectos durante el máster y en la $2^{a}$ parte, se trata de valorar la importancia que pueden tener tales aspectos en el proceso de formación inicial docente o la atención que la universidad debería prestar a tales temas para mejorar la acción formativa del máster en el futuro. Del total de los 30 ítems que recoge el cuestionario, este estudio se refiere a 11 de ellas: competencias establecidas para el Prácticum (C) del Máster FPES. Antes de su edición definitiva el cuestionario fue revisado por varios investigadores expertos, que sugirieron diversas modificaciones y ayudaron a establecer su redacción definitiva, tal como aparece en la Tabla 1. 
Tabla 1.

Variables relacionadas con las competencias del prácticum

\begin{tabular}{|l|c|c|}
\hline \multirow{2}{*}{$\begin{array}{l}\text { Enunciados de ítems de la sección C } \\
\text { (Valoración en una escala de 1 a 5) }\end{array}$} & $\begin{array}{c}\text { Nivel de } \\
\text { desarrollo }\end{array}$ & $\begin{array}{c}\text { Importancia } \\
\text { formativa }\end{array}$ \\
\cline { 2 - 4 } $\begin{array}{l}\text { C1. Adquirir experiencia en planificación, docencia y evaluación } \\
\text { de las materias correspondientes a la especialización }\end{array}$ & $\mathrm{Cn} 1$ & $\mathrm{Ci} 1$ \\
\hline $\begin{array}{l}\text { C2. Acreditar un buen dominio de la expresión oral y escrita en } \\
\text { la práctica docente }\end{array}$ & $\mathrm{Cn} 2$ & $\mathrm{Ci} 2$ \\
\hline $\begin{array}{l}\text { C3. Dominar destrezas y habilidades sociales necesarias para } \\
\text { fomentar un clima que facilite aprendizaje y convivencia }\end{array}$ & $\mathrm{Cn} 3$ & $\mathrm{Ci} 3$ \\
\hline $\begin{array}{l}\text { C4. Participar en propuestas de mejora en los distintos ámbitos } \\
\text { de actuación a partir de la reflexión basada en la práctica }\end{array}$ & $\mathrm{Cn} 4$ & $\mathrm{Ci} 4$ \\
\hline $\begin{array}{l}\text { C5. Para la formación profesional, conocer la tipología } \\
\text { empresarial correspondiente a los sectores productivos y } \\
\text { comprender los sistemas organizativos más comunes en las } \\
\text { empresas }\end{array}$ & $\mathrm{Cn} 5$ & $\mathrm{Ci5}$ \\
\hline $\begin{array}{l}\text { C6. Respecto a la orientación, ejercitarse en la evaluación } \\
\text { psicopedagógica, el asesoramiento a otros profesionales de la } \\
\text { educación, a los estudiantes y a las familias }\end{array}$ & $\mathrm{Cn} 6$ & $\mathrm{Ci} 6$ \\
\hline $\begin{array}{l}\text { C7. Valorar el papel de la cultura organizativa de cada centro y } \\
\text { conocer las funciones de los diversos elementos que lo integran }\end{array}$ & $\mathrm{Cn} 7$ & $\mathrm{Ci} 7$ \\
\hline $\begin{array}{l}\text { C8. Contrastar la visión personal de la enseñanza con resto de } \\
\text { profesionales del centro para tomar decisiones conjuntas }\end{array}$ & $\mathrm{Cn} 8$ & $\mathrm{Ci} 8$ \\
\hline $\begin{array}{l}\text { C9. Planificar el proceso de enseñanza en el área, diseñando } \\
\text { materiales didácticos y tareas educativas }\end{array}$ & $\mathrm{Cn} 9$ & $\mathrm{Ci} 9$ \\
\hline $\begin{array}{l}\text { C10. Desarrollar procesos de interacción y de comunicación } \\
\text { efectiva en el aula, acreditando un buen dominio de la } \\
\text { expresión oral y escrita en la práctica docente }\end{array}$ & $\mathrm{Cn} 10$ & $\mathrm{Ci} 10$ \\
\hline $\begin{array}{l}\text { C11. Analizar los resultados de la evaluación y extraer } \\
\text { conclusiones que ayuden a mejorar los procesos de enseñanza } \\
\text { yaprendizaje }\end{array}$ & $\mathrm{Cn} 11$ & $\mathrm{Ci11}$ \\
\hline
\end{tabular}

\section{Técnicas de análisis de datos}

Los datos recogidos con el cuestionario se han codificado como datos numéricos de una escala ordinal y con ayuda del paquete informático SPSS V.20 se han aplicado los tratamientos estadísticos similares a los del estudio anterior. A continuación se citan los principales tipos de análisis realizados sobre tales datos en relación con los fines de este estudio: a) Análisis descriptivo de frecuencias y valores medios para conocer el grado de acuerdo (o desacuerdo) con las diferentes proposiciones recogidas en los ítems del cuestionario; b) Prueba Alfa de Crombach para conocer la fiabilidad del cuestionario completo y de cada una de sus partes; c) Prueba de Mann-Witney para comparar valores medios en las diferentes variables del cuestionario entre las diversas submuestras que integran el conjunto global de sujetos encuestados; d) Análisis de conglomerados y escalamiento multidimensional para identificar las agrupaciones de sujetos que muestran un conjunto de opiniones similares en las diversas subescalas que integran el cuestionario; 
e) Prueba de Kolmogorov-Smirnov para comparar valores medios entre variables relacionadas con el nivel de desarrollo y la importancia formativa atribuida a las diferentes competencias.

El cuestionario en su globalidad presenta un elevado grado de consistencia interna (coeficiente alfa igual a 0,956) y, en particular, la Sección C del cuestionario (cuyos datos se analizan en este estudio) muestra un valor alfa igual a 0,911 en el conjunto de ítems relativos al nivel de desarrollo de competencias del Prácticum (Cn) y un valor 0,899 en el conjunto de ítems relativos a la importancia atribuida a tales competencias ( $\mathrm{Ci}$ ) y un valor de 0.911 .

Para comprobar que el cuestionario es fiable y que proporciona resultados similares en diferentes sub-muestras de una misma población, se ha realizado un estudio de comparación de medias entre los grupos de sujetos correspondientes a las diversas áreas de conocimiento. Se ha utilizado la prueba no paramétrica $U$ de Mann-Witney ( $M-W)$, como medida de contraste, ya que trabajamos con variables de carácter ordinal. No se han encontrado apenas diferencias significativas entre las diferentes submuestras de participantes, para la mayoría de los ítems del cuestionario, por lo que consideramos que suficientemente fiable (Serrano, 2013).

\section{Resultados}

\section{Nivel de desarrollo de las competencias del prácticum}

Las once competencias específicas del módulo Prácticum del máster FPES se incluyen en la sección C del cuestionario global (Serrano, 2013) y hemos asignado el código Cn a las variables relativas a la valoración realizada por los sujetos encuestados en este estudio sobre el nivel de desarrollo de tales competencias. En primer lugar se ha realizado un estudio descriptivo de tales variables que nos ha permitido conocer el valor medio y otros estadísticos descriptivos de tales variables, a nivel de toda la muestra. También se han se han obtenido las frecuencias absolutas y relativas (porcentajes) en las cinco categorías de respuesta de la escala Líkert empleada para medir el grado de acuerdo con cada proposición ( $1=$ nada, 2 = poco, $3=$ intermedio, 4 = bastante; $5=$ mucho). En la tabla 2 se recogen los resultados de este primer análisis. 
Tabla 2.

Datos descriptivos relativos al nivel de desarrollo en las competencias del practicum

\begin{tabular}{|c|c|c|c|c|c|c|c|c|c|c|c|}
\hline \multirow{2}{*}{ Valores } & \multicolumn{11}{|c|}{ Frecuencias relativas (\%) } \\
\hline & C1n & $\mathrm{C}_{2 n} \mathrm{n}$ & $C_{3 n}$ & $\mathrm{C}_{4} \mathrm{n}$ & $C_{5 n}$ & C6n & $C_{7 n}$ & C8n & C9n & C10n & $C_{11 n}$ \\
\hline 1 (Nada) & 2,5 & 4,5 & 3,4 & 5,7 & 19,0 & 8 & 2,8 & 9 & 3,1 & 2,8 & 4,5 \\
\hline 2 (Poco) & 9,1 & 11,6 & 13,0 & 21,0 & 21,2 & 21,0 & 5,9 & 13,9 & 7,4 & 11,6 & 11,3 \\
\hline 3 (Medio) & 34,6 & 33,7 & 31,7 & 32,6 & 30,3 & 29,7 & 32,6 & 32,3 & 24,4 & 28,9 & 30,3 \\
\hline $\begin{array}{c}4 \\
\text { (Bastante) }\end{array}$ & 33,7 & 35,4 & 37,4 & 31,4 & 21,0 & 29,2 & 37,1 & 32,0 & 34,8 & 36,3 & 31,7 \\
\hline \multirow[t]{2}{*}{5 (Mucho) } & 20,1 & 14,7 & 14,4 & 9,3 & 8,5 & 11,6 & 21,5 & 15,9 & 30,3 & 20,4 & 22,1 \\
\hline & \multicolumn{11}{|c|}{ Otros datos descriptivos } \\
\hline Media & 3,60 & 3,44 & 3,46 & 3,18 & 2,79 & 3,14 & 3,69 & 3,38 & 3,82 & 3,62 & 3,56 \\
\hline $\begin{array}{l}\text { Desv. } \\
\text { típica }\end{array}$ & 990 & 1,024 & 1,003 & 1,047 & 1,217 & 1,135 & ,968 & 1,091 & 1,045 & 1,026 & 1,091 \\
\hline
\end{tabular}

Si ordenamos tales ítems en función del valor medio otorgado al nivel de desarrollo podemos observar varios tipos de competencias del prácticum con algunas diferencias.

\section{- Competencias más desarrolladas:}

(C9n) Planificar el proceso de enseñanza en el área, diseñando materiales didácticos y tareas educativas $(3,82)$.

(C7n) Valorar el papel de la cultura organizativa de cada centro y conocer las funciones de los diversos elementos que lo integran $(3,69)$.

(C10n) Desarrollar procesos de interacción y de comunicación efectiva en el aula, acreditando un buen dominio de la expresión oral y escrita en la práctica docente $(3,62)$.

(C1n) Adquirir experiencia en planificación, docencia y evaluación de las materias correspondientes a la especialización (3,60).

(C11n) Analizar los resultados de la evaluación y extraer conclusiones que ayuden a mejorar los procesos de enseñanza y aprendizaje $(3,56)$.

(C3n) Dominar destrezas y habilidades sociales necesarias para fomentar un clima que facilite aprendizaje y convivencia $(3,46)$.

(C2n) Acreditar un buen dominio de la expresión oral y escrita en la práctica docente $(3,44)$.

(C8n) Contrastar la visión personal de la enseñanza con resto de profesionales del centro para tomar decisiones conjuntas $(3,38)$. 


\section{- Competencias menos desarrolladas:}

(C4n) Participar en propuestas de mejora en los distintos ámbitos de actuación a partir de la reflexión basada en la práctica $(3,18)$.

(C6n) Respecto a la orientación, ejercitarse en la evaluación psicopedagógica, el asesoramiento a otros profesionales de la educación, a los estudiantes y a las familias $(3,14)$.

(C5n) Para la formación profesional, conocer la tipología empresarial correspondiente a los sectores productivos y comprender los sistemas organizativos más comunes en las empresas $(2,79)$.

Entre los tratamientos estadísticos relacionados con estas variables podemos indicar que se ha realizado un análisis factorial sobre el conjunto de tales variables y hemos observado la existencia de un factor principal que agrupa a la gran mayoría de ellas. Al realizar después un análisis de conglomerados (Clúster) de tales variables apreciamos la existencia de un clúster principal, que agrupa a todas estas variables, quedando un poco desligada del clúster principal la variable $\mathrm{C}_{4} \mathrm{n}$. El escalamiento multidimensional también corrobora este resultado, ya que casi todas las variables del conglomerado principal aparecen muy juntas en el mapa bidimensional que se obtiene y la variable C4n queda un poco más alejada del núcleo central. También se ha realizado un análisis de correlación (determinando los coeficientes de correlación "Rho de Spearman") y observamos que las asociaciones entre tales variables son altas y significativas (al nivel p>0'01) en todos los casos, de modo que podemos considerar las ideas agrupadas en esta subescala presentan un alto grado de consistencia interna (Serrano, 2013).

Importancia formativa de las competencias específicas del prácticum

Tabla 3 .

Datos descriptivos relativos a la importancia formativa de las competencias del practicum

\begin{tabular}{|c|c|c|c|c|c|c|c|c|c|c|c|}
\hline \multirow{2}{*}{ Valores } & \multicolumn{11}{|c|}{ Frecuencias relativas (\%) } \\
\hline & $\mathrm{C}_{11} \mathrm{i}$ & $\mathrm{C}_{2} \mathbf{i}$ & $\mathrm{C}_{3} \mathbf{i}$ & $\mathrm{C}_{4} \mathrm{i}$ & $\mathrm{C}_{5} \mathbf{i}$ & C6i & $C_{7} \mathbf{i}$ & $\mathrm{C} 8 \mathrm{i}$ & C9i & C10i & C11i \\
\hline 1 (Nada) & 6 & ,8 & ,6 & ,8 & 4,8 & 1,4 & 1,7 & 6 & 8 & 8 & 1,1 \\
\hline 2 (Poco) & 2,0 & 2,3 & 1,7 & 2,8 & 8,2 & 3,1 & 2,3 & 3,1 & 2,8 & 2,5 & 2,5 \\
\hline 3 (Medio) & 6,8 & 10,2 & 6,2 & 18,4 & 26,9 & 16,7 & 13,9 & 13,3 & 6,8 & 6,8 & 7,6 \\
\hline $\begin{array}{c}4 \\
\text { (Bastante) }\end{array}$ & 23,2 & 33,7 & 29,5 & 42,2 & 30,6 & 35,7 & 43,9 & 41,9 & 18,4 & 28,6 & 25,2 \\
\hline \multirow[t]{2}{*}{5 (Mucho) } & 67,4 & 53,0 & 62,0 & 35,7 & 29,5 & 43,1 & 38,2 & 41,1 & 71,1 & 61,2 & 63,5 \\
\hline & \multicolumn{11}{|c|}{ Otros datos descriptivos } \\
\hline Media & 4,52 & 4,36 & 4,53 & 4,13 & 3,72 & 4,16 & 4,15 & 4,20 & 4,49 & 4,50 & 4,47 \\
\hline $\begin{array}{l}\text { Desv. } \\
\text { típica }\end{array}$ & ,756 & ,821 & ,739 & 1,940 & 1,118 & ,909 & ,863 & ,826 & ,810 & 801 & ,833 \\
\hline
\end{tabular}

Si ordenamos tales ítems en función del valor medio otorgado a la importancia formativa podemos considerar varios tipos de competencias. 


\section{- Competencias más importantes:}

(C3i) Dominar destrezas y habilidades sociales necesarias para fomentar un clima que facilite aprendizaje y convivencia $(4,53)$.

(C1i) Adquirir experiencia en planificación, docencia y evaluación de las materias correspondientes a la especialización $(4,52)$.

(C10i) Desarrollar procesos de interacción y de comunicación efectiva en el aula, acreditando un buen dominio de la expresión oral y escrita en la práctica docente $(4,50)$.

(C9i) Planificar el proceso de enseñanza en el área, diseñando materiales didácticos y tareas educativas $(4,49)$.

(C11i) Analizar los resultados de la evaluación y extraer conclusiones que ayuden a mejorar los procesos de enseñanza y aprendizaje $(4,47)$.

(C2i) Acreditar un buen dominio de la expresión oral y escrita en la práctica docente $(4,36)$.

\section{- Competencias de importancia intermedia:}

(C8i) Contrastar la visión personal de la enseñanza con resto de profesionales del centro para tomar decisiones conjuntas $(4,20)$.

(C6i) Respecto a la orientación, ejercitarse en la evaluación psicopedagógica, el asesoramiento a otros profesionales de la educación, a los estudiantes y a las familias $(4,16)$.

(C7i) Valorar el papel de la cultura organizativa de cada centro y conocer las funciones de los diversos elementos que lo integran $(4,15)$.

(C4i) Participar en propuestas de mejora en los distintos ámbitos de actuación a partir de la reflexión basada en la práctica $(4,13)$.

\section{- Competencia menos relevante}

(C5i) Para la formación profesional, conocer la tipología empresarial correspondiente a los sectores productivos y comprender los sistemas organizativos más comunes en las empresas (3,72).

En el conjunto de estas variables se han realizado similares tratamientos estadísticos (análisis factorial, análisis de conglomerados, escalamiento multidimensional y análisis de correlaciones), encontrando de nuevo la existencia de un factor principal, que agrupa a la gran mayoría de variables de esta sección. El escalamiento multidimensional y análisis de conglomerados muestran la existencia de un núcleo principal, que agrupa a casi todas estas variables, quedando un poco desligadas del clúster principal las variables C4i y C5i.

\section{Contraste entre valoración e importancia de las competencias del practicum}

Por último vamos a tratar de profundizar en el análisis de las diferencias entre las opiniones de los participantes sobre el nivel de desarrollo de las competencias del módulo Prácticum (Cn) y el grado de importancia formativa que les conceden a tales competencias (Ci), comenzando por estudiar las diferencias que se observan en el análisis de frecuencias. 
Al tratarse de una escala de valoración de cinco niveles se ha realizado una agrupación de frecuencias por los extremos, que permite una interpretación más sencilla de resultados, considerando los niveles de respuesta siguientes: I (nada o poco de acuerdo), II (posición intermedia) y III (bastante o muy de acuerdo).

En la tabla 4 se muestran los resultados comparativos de las frecuencias agrupadas en ambos tipos de variables. Se puede observar que el nivel de desarrollo de competencias del Prácticum alcanza un valor medio moderado en la mayor parte de los ítems, con porcentajes de acuerdo comprendidos entre el $65 \%$ y el 40\%, excepto en el ítem C5 que es bastante bajo (29\%). También se observa que la importancia formativa atribuida a tales competencias alcanza una valoración bastante más alta en todos los ítems, ya que se obtienen porcentajes de amplio acuerdo comprendidos entre el $91 \%$ y el $60 \%$. Esto indica que las expectativas sobre las competencias a desarrollar en el módulo Prácticum del máster quedan muy por encima de los logros alcanzados en tales aspectos.

Los resultados anteriores muestran que los estudiantes del máster FPES de la Universidad de Córdoba valoran de forma moderada el nivel de desarrollo de las competencias del Prácticum, durante los dos primeros años de su implantación, pero atribuyen una importancia grande a tales aspectos como elementos que pueden contribuir a la mejora de la formación inicial del profesorado de secundaria. Por ello hemos realizado un estudio comparativo más profundo de tales datos de la sección $C$ del cuestionario, con objeto de valorar si las diferencias entre tales datos son significativas y extraer conclusiones que puedan servir para mejorar el proceso formativo en futuras ediciones del máster FPES.

Tabla 4 .

Comparación de frecuencias relativas correspondientes a la valoración del nivel de desarrollo y la importancia asignada a las competencias del Prácticum

\begin{tabular}{|c|ccc|c|ccc|}
\hline $\begin{array}{c}\text { Nivel de } \\
\text { desarrollo }\end{array}$ & \multicolumn{2}{|c|}{ Porcentajes (por niveles) } & \multicolumn{3}{|c|}{ Importancia } & \multicolumn{3}{|c|}{ Porcentajes (por niveles) } \\
\hline Cn1 & 11,6 & (II) & (III) & formativa & (I) & (II) & (III) \\
\hline Cn2 & 16,1 & 33,6 & 53,8 & Ci1 & 2,5 & 6,8 & 90,7 \\
\hline Cn3 & 16,4 & 31,7 & 50,1 & Ci2 & 3,1 & 10,2 & 86,7 \\
\hline Cn4 & 26,6 & 32,6 & 40,8 & Ci3 & 2,3 & 6,2 & 91,5 \\
\hline Cn5 & 40,2 & 30,3 & 29,5 & Ci5 & 3,7 & 18,4 & 77,9 \\
\hline Cn6 & 29,5 & 29,9 & 40,6 & Ci6 & 4,5 & 26,9 & 60,1 \\
\hline Cn7 & 8,8 & 32,6 & 58,6 & Ci7 & 4,0 & 13,7 & 78,8 \\
\hline Cn8 & 19,8 & 32,3 & 47,9 & Ci8 & 3,7 & 13,3 & 83,2 \\
\hline Cn9 & 10,5 & 24,4 & 65,2 & Ci9 & 3,7 & 6,8 & 89,5 \\
\hline Cn10 & 14,4 & 28,9 & 56,7 & Ci10 & 3,4 & 6,8 & 89,8 \\
\hline Cn11 & 15,9 & 30,5 & 53,6 & Ci11 & 3,7 & 7,6 & 88,7
\end{tabular}

En la tabla 5 se muestran los resultados obtenidos, mediante la prueba $Z$ de Kolmogorov-Smirnov, al comparar el nivel de desarrollo y la importancia asignada a las 11 competencias del Prácticum. Se muestran también los valores medios de cada competencia en la primera y segunda parte del cuestionario, la diferencia entre valores medios de tales ítems en ambas partes (Dni) y la significatividad ( $p$ ) de las pruebas de contraste. Se puede observar que existen diferencias muy significativas (al nivel $p<0,001$ ) en todos los ítems de 
la sección $\mathrm{C}$ al comparar el nivel ( $\mathrm{n}$ ) de desarrollo de competencias con la importancia (i) formativa atribuida a cada una de estas competencias.

Tabla 5.

Contraste de valores medios entre nivel de desarrollo e importancia de las competencias

\begin{tabular}{|c|c|c|c|cc|}
\hline \multirow{2}{*}{ íTEMS } & \multicolumn{2}{|c|}{ Valores medios } & \multirow{2}{*}{ Diferencia } & \multicolumn{2}{c|}{ Prueba de contraste } \\
\cline { 2 - 3 } & Nivel (n) & Importancia (i) & (Dni) & Z (K-S) & p \\
\hline C1 & 3,60 & 4,55 &, 952 & 6,285 &, 000 \\
\hline C2 & 3,44 & 4,36 &, 915 & 5,081 &, 000 \\
\hline C3 & 3,46 & 4,51 & 1,042 & 6,323 &, 000 \\
\hline C4 & 3,18 & 4,09 &, 912 & 4,930 &, 000 \\
\hline C5 & 2,79 & 3,72 &, 929 & 4,065 &, 000 \\
\hline C6 & 3,14 & 4,16 & 1,014 & 5,043 &, 000 \\
\hline C7 & 3,69 & 4,15 &, 462 & 3,124 &, 000 \\
\hline C8 & 3,38 & 4,20 &, 819 & 4,667 &, 000 \\
\hline C9 & 3,82 & 4,56 &, 742 & 5,420 &, 000 \\
\hline C10 & 3,60 & 4,47 &, 870 & 5,420 &, 000 \\
\hline C11 & 3,56 & 4,47 &, 918 & 5,495 &, 000 \\
\hline
\end{tabular}

A partir de los datos de la tabla 5 se puede analizar la diferencia entre la importancia que los sujetos conceden a las competencias del Prácticum y el nivel de desarrollo alcanzado en cada una de estas competencias. También podemos distinguir diferentes situaciones, según el valor de la diferencia entre tales aspectos (Dni):

- Competencias generales con una diferencia muy elevada entre importancia y nivel de desarrollo

(C3) Dominar destrezas y habilidades sociales necesarias para fomentar un clima que facilite aprendizaje y convivencia (Dni: 1,042).

(C6) Respecto a la orientación, ejercitarse en la evaluación psicopedagógica, el asesoramiento a otros profesionales de la educación, a los estudiantes y a las familias (Dni: 1,014).

(C1) Adquirir experiencia en planificación, docencia y evaluación de las materias correspondientes a la especialización (Dni: 0,952).

(C5) Para la formación profesional, conocer la tipología empresarial correspondiente a los sectores productivos y comprender los sistemas organizativos más comunes en las empresas (Dni: 0,929).

(C11) Analizar los resultados de la evaluación y extraer conclusiones que ayuden a mejorar los procesos de enseñanza y aprendizaje (Dni: 0,918).

(C2) Acreditar un buen dominio de la expresión oral y escrita en la práctica docente (Dni: $0,915)$. 
(C7) Valorar el papel de la cultura organizativa de cada centro y conocer las funciones de los diversos elementos que lo integran (Dni: 0,912).

- Competencias del prácticum con una diferencia elevada entre importancia y nivel de desarrollo

(C10) Desarrollar procesos de interacción y de comunicación efectiva en el aula, acreditando un buen dominio de la expresión oral y escrita en la práctica docente (Dni: 0,870).

(C8) Contrastar la visión personal de la enseñanza con resto de profesionales del centro para tomar decisiones conjuntas (Dni: 0,819).

- Competencias del prácticum que presentan menos diferencia entre importancia y nivel de desarrollo

(C9) Planificar el proceso de enseñanza en el área, diseñando materiales didácticos y tareas educativas (Dni: 0,742).

(C4) Participar en propuestas de mejora en los distintos ámbitos de actuación a partir de la reflexión basada en la práctica (Dni: 0,462).

\section{Discusión y conclusiones}

La implantación y puesta en marcha del Máster de formación del profesorado de enseñanza secundaria (MFPES) hace sólo unos años ha fomentado el interés por la investigación educativa en esta temática. Por ello estamos desarrollando un proyecto de renovación del proceso de formación inicial docente, centrado en la exploración del pensamiento inicial del alumnado del máster FPES para conocer sus intereses profesionales, actitudes hacia la docencia y conocimientos o ideas previas sobre diversos aspectos de la educación (Pontes y Serrano, 2010; Pontes, Serrano y Poyato, 2013). En este trabajo hemos tratado de analizar las expectativas de nuestro alumnado en torno a las competencias profesionales que esperan alcanzar durante la fase de prácticas en centros de enseñanza secundaria y se han comparado tales expectativas con la valoración crítica del nivel de desarrollo que alcanzan tales competencias.

Entre los aspectos a destacar debemos comenzar indicando que se aprecian diferencias notables, entre la importancia atribuida a las competencias específicas del Prácticum y el nivel de desarrollo alcanzado en tales aspectos, tras el proceso de formación inicial del profesorado de secundaria. En particular se ha observado que los futuros docentes consideran que han adquirido un nivel de formación moderadamente satisfactorio tras haber cursado el Máster FPES (Pontes y Serrano, 2008; Cano, Orejudo y Cortés, 2012; Torrego, 2013). Sin embargo se aprecia que sus expectativas formativas eran mayores, ya que la importancia atribuida a las diversas competencias del prácticum del Máster FPES es más elevada en general (Hascher, Cocard y Moser, 2004; González Sanmamed, 2009; Mendoza, 2013). Se deduce, por tanto, que la formación inicial docente recibida por el futuro profesorado de secundaria, durante los primeros años de experimentación del máster podría mejorar bastante, en lo relativo al Prácticum (Cebrián, 2011; Zabalza, 2011; Albert, Fuentes y Palos, 2012). La diferencia apreciada entre expectativas y logros alcanzados por los estudiantes en la FIPS puede ser un indicador útil para identificar fortalezas y debilidades del Máster FPES, que permitan posteriormente trabajar 
en reducir los puntos débiles y mejorar la formación inicial docente (González y Maldonado, 2010).

Entre los resultados obtenidos, encontramos que las competencias mejor valoradas por los estudiantes y las estudiantes -en cuanto a su grado de desarrollo- se relacionan, sobre todo, con la planificación de los procesos educativos $(65,2 \%)$ y disciplinares $(53,8 \%)$. Resultados similares a los obtenidos en la investigación de Manso y Martín (2013). Por otro lado, también destacan el dominio de habilidades y destrezas que mejoren la convivencia $(51,8 \%)$ (Pontes y Serrano, 2008) y los procesos de interacción y comunicación afectiva en el aula (56,7\%), lo que Álvarez (2010) denomina "competencias complementarias". Es destacable como el desarrollo de competencias vinculadas a la cultura organizativa del centro es valorada muy positivamente $(58,6 \%)$ por los y las estudiantes del Máster FPES y muy en la línea de las aportaciones de autores como Coll (2010), Feito (2010) y Pérez, (2010). El logro de tales competencias se puede relacionar con el desarrollo de un pensamiento docente reflexivo y centrado en los procesos de enseñanza-aprendizaje (Michavila y Esteve, 2011).

La competencia menos valorada y por lo tanto, una de las principales debilidades del proceso de formación se corresponde con la formación profesional (29,5\%). Consideramos este dato poco significativo porque es una minoría el alumnado que realiza su periodo de prácticas integrado en ciclos formativos (Puentes, 2013). Por otro lado, la conexión con el asesoramiento a otros profesionales de la educación, la familia y los estudiantes es otra de las competencias con un grado de adquisición bajo (40,6\%). Esto nos lleva a pensar que las funciones de tutoría, orientación y asesoramiento psicopedagógico son un fleco en la formación inicial docente, como ya apuntaban Pérez-Valiente (2011) y Cid, Pérez y Sarmiento (2011).

Por otro lado, los futuros docentes manifiestan, según el -grado de importanciaatribuido a las competencias del prácticum unos valores medios bastante elevados en la mayoría de los ítems, que apuntan hacia la importancia que los estudiantes del máster FPES conceden a estos aspectos para la formación docente.

Podemos observar que existe un patrón similar en la valoración del grado de importancia formativa y del nivel de desarrollo alcanzado, ya que existe bastante paralelismo entre las competencias mejor adquiridas y las más importantes de acuerdo a las opiniones de los encuestados, y viceversa, las principales debilidades se enmarcan dentro de las mismas competencias con un grado de adquisición menor y con un nivel de importancia inferior. Podemos pensar que esta relación no es arbitraria, sino que pone de manifiesto las principales debilidades y fortalezas del Máster FPES, en sus primeros años de implantación, lo que nos puede ayudar a formular una propuesta fundamentada de mejoras en la FIPS.

Estos resultados, si se tienen en cuenta que corresponden a los primeros y problemáticos años de implantación del máster nos parecen moderadamente positivos, ya que apuntan hacia algunas de las potenciales fortalezas del Prácticum, en las que habrá que seguir mejorando posteriormente (Sarmiento, Cid y Pérez, 2011; Zabalza, 2011; Morales, Narváez y Morales, 2012).

Finalmente debemos señalar que los resultados del estudio deben ser tomados con cautela debido a sus posibles limitaciones, relacionadas con el tamaño de la muestra y la 
naturaleza del instrumento de recogida. Sin embargo, a pesar de tales limitaciones consideramos que la información aportada en este estudio puede servir de base para desarrollar en el futuro análisis más complejos y procesos de investigación más minuciosos en torno a las expectativas y al desarrollo de las competencias docentes en el módulo Prácticum del máster FPES.

\section{Referencias}

Albert, J.M., Fuentes, C. Y Palos, J. (2012). "Formación inicial del profesorado y el Prácticum de secundaria”. Iber: Didáctica de las ciencias sociales, geografía e historia, 72, 65-70.

Álvarez, D., Rodríguez, C., González, P., Núñez, J.C. Y Álvarez, L. (2010). “La formación de los futuros docentes frente a la violencia escolar". Revista de Psicodidáctica, 15 (1), $35-56$.

Armengol, C., Castro, D., Jariot, M., Massot, M. Y Sala, J. (2011). "El Prácticum en el Espacio Europeo de Educación Superior (EEES): mapa de competencias del profesional de la educación". Revista de Educación, 354 (enero-abril), 71-98.

Bolívar, A. (2006). La identidad profesional del profesorado de secundaria: Crisis y reconstrucción. Archidona: Aljibe.

Cano, J., Orejudo, S. Y Cortés, A. (2012). "La formación inicial del profesorado de secundaria: primera investigación en el desarrollo del prácticum en la Universidad de Zaragoza”. REIFOP, 15 (3), 121-132.

Cebrián, M. (2011). "Evaluación formativa del Prácticum con e-portfolios y e-rúbricas". En Raposo, M.; Martínez M.E.; Muñoz, P.C.; Abellás, A y Otero, J.C. (coords.) Evaluación y supervisión del Prácticum: el compromiso con la calidad de las prácticas (pp. 143149). Santiago de Compostela: Andavira.

Cid, A., Pérez, A. Y Sarmiento, J.A. (2011). "La tutoría en el Prácticum. Revisión de la literatura”. Revista de Educación, 354 (enero-abril), 127-154.

Coll, C. (2010). Desarrollo, aprendizaje y enseñanza en la educación secundaria. Barcelona: Graó.

Feito, R. (2010). Sociología de la educación secundaria. Barcelona: Graó.

Garín, J. (2011). Formación de profesores basada en competencias. Bordón, 63 (1), 77-92.

González, I. Y Maldonado, A. (2010). "Una mirada evaluadora del Máster desde las ciencias de la educación. Hacia la gobernanza universitaria”. En C. GONZÁLEZ, (Coord.), El nuevo profesor de Secundaria. La formación inicial docente en el marco del Espacio Europeo de Educación Superior. Barcelona: Grao, 335-355

González Sanmamed, M. (2009). "Una nueva oportunidad para la formación inicial del profesorado de Educación Secundaria”. Revista de Educación, 350, 57-78.

González Sanmamed, M. Y Abeledo, E. (2011). "El Prácticum en el aprendizaje de la profesión de docente". Revista de educación, 354, 47-70.

Gutiérrez, J. Y Otros. (2003). El Practicum en la formación inicial del Profesorado de Magisterio y Educación Secundaria: Avances de investigación, Fundamentos y Programas de Formación. Granada: Universidad de Granada. 
Gutiérrez, J.M. (2011). La formación inicial del profesorado de Secundaria. Del CAP al máster. CEE: Participación Educativa, 17, 96-107.

Hascher, T., Cocard, Y., Y Moser, P. (2004). "Forget about theory practice is all? Student teacher's learning in Prácticum". Teacher and Teaching: Theory and Practice, 10 (6), 623-637.

Manso, J. Y Martín, E. (2013). "Valoración del Máster de Formación de Profesorado de Educación Secundaria: estudio de casos en dos universidades". Revista de Educación, 364 (abril-junio), 145-169.

Martín, C., Prieto, Y. Y Lupión, T (2014). Profesorado de ciencias en formación inicial ante la enseñanza y el aprendizaje de las ciencias: ¿perfil innovador o tradicional? Revista Electrónica Interuniversitaria de Formación del Profesorado, 17(1), 149-163.

Mellado, V., Blanco, L. y Ruiz, C. (1999). Aprender a enseñar ciencias experimentales en la formación inicial de profesorado. Badajoz: ICE de la Universidad de Extremadura.

Mendoza, M. (2013). Adquisición y desarrollo de competencias profesionales en el prácticum de los grados de magisterio: Estudio empírico desde la perspectiva de sus estudiantes (Tesis doctoral), Universidad Complutense de Madrid, Madrid, España. Recuperado de http:// eprints.ucm.es/20566/1/T34368.pdf

Michavila, F. Y Esteve, F. (2011). "La llegada a la universidad: ¿oportunidad o amenaza?” Participación educativa, 17, 69-85.

Morales, F.M., Narváez, M. Y Morales, A. (2012). “Percepciones de los futuros docentes sobre la cultura organizacional de los centros de prácticas externas de Educación Secundaria". Revista Teoría de la Educación: Educación y Cultura en la Sociedad de la Información, 13 (2), 173-199.

Pérez, A. (2010). Aprender a enseñar en la práctica: Procesos de innovación y prácticas de formación en la educación secundaria. Barcelona: Graó.

Pérez-Valiente, P-J. (2011). "Prácticum de calidad para el Máster docente de Secundaria". Participación Educativa, 16, 114-121.

Perrenoud, P. (2001). Desarrollar la práctica reflexiva en el oficio de enseñar. Barcelona: Graó.

Pontes, A. Y Serrano, R. (2008). "Reflexiones sobre la docencia tras el prácticum de la formación inicial del profesorado de enseñanza secundaria". I Congreso Internacional sobre Profesorado Principiante e Inserción Profesional a la Docencia. Universidad de Sevilla.

Pontes, A. Y Serrano, R. (2010). "La formación inicial en un contexto de cambio". En González, I. El nuevo profesor de secundaria. La formación docente en el marco del Espacio Europeo de Educación Superior. Barcelona: Graó.

Pontes, A., Serrano, R. Y Poyato, F.J. (2013). Concepciones y motivaciones sobre el Desarrollo Profesional Docente en la Formación Inicial del Profesorado de Secundaria. Revista eureka sobre Enseñanza y Divulgación de las Ciencias, 10, 533-551.

Puentes, R. (2013). "El modelo de formación del profesorado de educación secundaria en España: el caso del Máster de la Universidad de Granada”. Informe Final de PostDoctorado. CAPES/ UGR.

Ramírez, S., Sánchez, C.A., García, A. Y Latorre, M.J. (2011). "El Prácticum en Educación Infantil, Primaria y Máster de Secundaria”. REDU: Revista de Docencia Universitaria, 9 (2), 257-259. 
Serrano, R. (2013). Identidad profesional, necesidades formativas y desarrollo de competencias docentes en la formación inicial del profesorado de secundaria. Tesis doctoral. Universidad de Córdoba.

Rodríguez, S. (2003). Nuevos retos y enfoques en la formación del profesorado universitario. Revista de Educación, 331, 67- 99

Rodríguez, M.J., Olmos, S. y Martínez, F. (2013). Evaluación de competencias informacionales en educación secundaria: un modelo causal. Cultura y Educación, 25(3), 361-373.

Rodríguez, C. Y Díez, E.J. (2014). Conocimiento y competencias básicas en la formación incial de maestras y maestros. Profesorado. Revista de curriculum y formación del profesortado, 18 (1). http://www.ugr.es/local/recfpro/rev181COL11.pdf

Sarmiento, J.A., Cid, A. Y Pérez, A. (2011). "Referencial, un instrumento para la evaluación y acreditación de las competencias desarrolladas a través del Prácticum del Máster de Secundaria”. REDU: Revista de Docencia Universitaria, 9 (2), 11-33.

Schön, D. A. (1992): La formación de profesionales reflexivos. Hacia un nuevo diseño de la enseñanza y el aprendizaje en las profesiones. Madrid: Paidós.

Solís, E., Porlán, R. Y Rivero, A. (2012). ¿Cómo representar el Conocimiento Curricular de los profesores de Ciencias y su evolución? Enseñanza de las Ciencias, 30 (3), 9-30.

Solís, E., Rivero, A. y Porlán, R. (2013). Expectativas y concepciones de los estudiantes del MAES en la especialidad de Ciencias. Revista Eureka sobre Enseñanza y Divulgación de las Ciencias, Vol. 10, No Extraordinario (FIPS)

Torrego, A. (2013). "El Prácticum desde dentro. Una visión del Prácticum del Máster de Secundaria de la Universidad de Valladolid a través de las opiniones de los estudiantes". En Santiago Castillo, Reflexiones, Análisis y Propuestas sobre la Formación del Profesorado de Educación Secundaria (Volumen 1)(402-432). Madrid: UNED.

Valdés , J. Y Bolívar, A. (2014). "La experiencia española de formación del profesorado: El Máster en Educación Secundaria”. Ensino Em Re-Vista, 21(1), 159-173.

Valverde, J., Garrido, M.C. Y Fernández, R. (2010). "Enseñar y aprender con tecnologías: un modelo teórico para las buenas prácticas con TIC". Teoría de la Educación. Educación y Cultura en la Sociedad de la Información, 11(3), 203-229.

Vázquez, P. y Ortega, J.L. (2011). Competencias Básicas. Desarrollo y evaluación en Educación Secundaria. Madrid: Wolters Kluwer.

Zabalza, Mi. A. (2004): Diarios de clase. Un instrumento de investigación y desarrollo profesional, Madrid: Narcea.

Zabalza, M.A. (2011). El Prácticum en la Formación Universitaria: Estado de la Cuestión. Revista de Educación, 354 (Enero-Abril), 21-43.

Zabalza, M.A. (2011). "Evaluar la calidad del Prácticum: una propuesta”. En Raposo, M.; Martínez M.E.; Muñoz, P.C.; Abellás, A y Otero, J.C. (coords.) Evaluación y supervisión del Prácticum: el compromiso con la calidad de las prácticas (101-128). Santiago de Compostela: Andavira. 